\title{
Investigating directed influences between activated brain areas in a motor-response task using fMRI
}

Citation for published version (APA):

Abler, B., Roebroeck, A. F., Goebel, R. W., Hose, A., Schonfeldt-Lecuona, C., Hole, G., \& Walter, H. (2006). Investigating directed influences between activated brain areas in a motor-response task using fMRI. Magnetic Resonance Imaging, 24(2), 181-185. https://doi.org/10.1016/j.mri.2005.10.022

Document status and date:

Published: 01/01/2006

DOI:

10.1016/j.mri.2005.10.022

Document Version:

Publisher's PDF, also known as Version of record

Document license:

Taverne

Please check the document version of this publication:

- A submitted manuscript is the version of the article upon submission and before peer-review. There can be important differences between the submitted version and the official published version of record.

People interested in the research are advised to contact the author for the final version of the publication, or visit the DOI to the publisher's website.

- The final author version and the galley proof are versions of the publication after peer review.

- The final published version features the final layout of the paper including the volume, issue and page numbers.

Link to publication

\footnotetext{
General rights rights.

- You may freely distribute the URL identifying the publication in the public portal. please follow below link for the End User Agreement:

www.umlib.nl/taverne-license

Take down policy

If you believe that this document breaches copyright please contact us at:

repository@maastrichtuniversity.nl

providing details and we will investigate your claim.
}

Copyright and moral rights for the publications made accessible in the public portal are retained by the authors and/or other copyright owners and it is a condition of accessing publications that users recognise and abide by the legal requirements associated with these

- Users may download and print one copy of any publication from the public portal for the purpose of private study or research.

- You may not further distribute the material or use it for any profit-making activity or commercial gain

If the publication is distributed under the terms of Article $25 \mathrm{fa}$ of the Dutch Copyright Act, indicated by the "Taverne" license above, 


\title{
Investigating directed influences between activated brain areas in a motor-response task using fMRI
}

\author{
Birgit Abler $^{\mathrm{a}, *}$, Alard Roebroeck ${ }^{\mathrm{b}}$, Rainer Goebel ${ }^{\mathrm{b}}$, Anett Höse ${ }^{\mathrm{a}}$, \\ Carlos Schönfeldt-Lecuona ${ }^{\mathrm{a}}$, Günter Hole ${ }^{\mathrm{c}}$, Henrik Walter ${ }^{\mathrm{a}}$ \\ a Department of Psychiatry, University of Ulm, 89075 Ulm, Germany \\ ${ }^{\mathrm{b}}$ Department of Cognitive Neuroscience, Maastricht University, 6229 Maastricht, The Netherlands \\ ${ }^{\mathrm{c}}$ Ravensburg, Germany \\ Received 10 March 2005; revised 15 October 2005; accepted 15 October 2005
}

\begin{abstract}
Localization of cognitive processes is a strength of functional neuroimaging. However, information about functional interactions between brain areas is crucial for a deeper understanding of brain function. We applied vector autoregressive modeling in the context of Granger causality as a method to analyze directed connectivity in a standard event-related fMRI study using a simple auditory-motor paradigm. The basic idea is to use temporal information in stochastic time series of a brain region in order to predict signal time courses in other brain regions. Thus, we predicted that the method should demonstrate causal influence of the auditory cortex and the supplementary motor area (SMA) on primary motor cortex.

Eleven right-handed healthy female subjects were instructed to press a ball with either their left or their right hand when hearing the command "left" or "right" in the scanner. Influence to the left motor cortex was found from bilateral auditory cortex as well as from the SMA in 9 of 11 subjects. Granger causality to the right motor cortex existed from bilateral auditory cortex in 5 and from SMA in 6 subjects. Granger causality to the SMA existed from right auditory cortex in 7 subjects and from left auditory cortex in 8 subjects.

Our findings in a simple task show that even under suboptimal circumstances (a relatively long TR of $2440 \mathrm{~ms}$ ), Granger causality can be a useful tool to explore effective connectivity. Temporally optimized scanning should increase that potential.

(C) 2006 Elsevier Inc. All rights reserved.
\end{abstract}

Keywords: Granger causality; Effective connectivity; fMRI; Auditory-motor paradigm

\section{Introduction}

Functional neuroimaging has been proved to be well suited to investigate the localization of certain cognitive functions in the brain. However, not only information about where cognitive processes take place in the brain, but also how they are implemented is crucial for a deeper understanding of brain function. Therefore, investigation of functional interactions as well as information about the direction of these interactions has become an issue in cognitive neurosciences in recent times. Directed interactions of brain areas are often referred to as effective connectivity, defined as influence of one neural system on another [1]. Several methods such as application of

\footnotetext{
* Corresponding author. Tel.: +49 0731500 41250; fax: +49 0731 50021549.

E-mail address: birgit.abler@uni-ulm.de (B. Abler).
}

covariance structural equation modeling [2] or nonlinear system identification techniques [3] have been proposed to investigate such effective connectivity. Recently, vector autoregressive modeling in the context of Granger causality has been introduced as a new method to analyze directed influences [4]. We applied the method in a standard eventrelated fMRI study with a simple auditory-motor design. The basic idea is to use temporal information in stochastic time series of a brain region in order to predict signal time courses in other brain regions. As a rule, causes precede their effects in time. Therefore, these predictions can be related to temporally directed influences rather than to only correlational relations between brain regions. In our paradigm, a simple motor action was triggered by an auditory command. Thus, we predicted that the method should demonstrate indirect causal influence of the auditory cortex on primary motor cortex and supplementary motor 
areas (SMAs). As the method is based on signal time series, the sampling of data points is an important issue. In this study, we used a design with a relatively long TR $(2440 \mathrm{~ms})$ and thus wide spacing of data points to investigate the limitations of the method under coarse temporal sampling.

\section{Materials and methods}

Written informed consent was obtained from 12 healthy right-handed female subjects aged 25 to 39 years (mean: 30.5 years) without any history of neurological or psychiatric disease. The study was approved by the local ethics committee.

\subsection{Task}

Subjects were instructed to press a ball with either their left or their right hand when hearing the command "left" or "right" ( $n=20$ each) in the scanner. The subject heard 20 commands of "right" and 20 commands of "left" from a taped voice in a pseudorandomized order through the headphones. The onsets of the commands were jittered between 5.2 and 19.2 s (mean: 10.1 s; S.D.: 3.9). Pressure exerted on the ball and reaction times defined as latency to maximal pressure were registered.

\section{2. fMRI acquisition}

A 1.5-T Siemens VISION whole-body scanner (Siemens, Erlangen, Germany) equipped with a head coil was used to acquire $\mathrm{T} 1$ anatomical volume images $(1 \times 1 \times 1 \mathrm{~mm}$ voxels $)$ and $\mathrm{T} 2 *$-weighted functional MR images. One hundred seventy-nine volumes of $29 \mathrm{~T} 2 *$-weighted echoplanar slices were recorded during one session. Image size was $64 \times$ 64 pixels, with an FoV of $192 \mathrm{~mm}$. Slice thickness was $4 \mathrm{~mm}$ with a 1-mm gap resulting in a voxel size of $3.5 \times 3.5 \times 4 \mathrm{~mm}$. Volumes were obtained at a TR of $2440 \mathrm{~ms}$ (TE $40 \mathrm{~ms}$ ).

\subsection{Data analysis}

fMRI data of the single subjects were analyzed eventrelated using the BrainVoyager 4.9 Software (BrainInnovation, R. Goebel, Maastricht, the Netherlands). The first four images of each functional scan were rejected to allow for $\mathrm{T} 2 *$ equilibration effects. Images were preprocessed including motion correction, slice scan time correction, high-pass filtering and removal of linear trends. After that, individual functional and anatomical data were coregistered and normalized into standard space [5]. Voxelwise analysis was performed using a general linear model for autocorrelated observations [6]. A standard model with regressors for left and right auditory command for motor movement convolved with a hemodynamic response function was calculated for each single subject. Statistical maps were thresholded at $P<.0001$ (uncorrected for multiple comparisons).

Activation foci found in the right and left motor cortex and the supplementary motor cortex (SMA) that passed this threshold were used to construct functional regions of interest (ROIs). One motor cortex ROI and one SMA ROI were constructed for each of the two regressors (left and right) resulting in four ROIs in each subject.

Custom software written in MATLAB (Mathworks, Natick, MA) was used to map Granger causality over the imaging volume for the selected ROIs for each subject individually. The method, described in detail in Roebroeck et al. [7] and Goebel et al. [4], treats the sequence of fMRI measurements at each voxel within the ROIs as a vector time series. It does not, like other methods of investigating directed connectivity, rely on a priori specification of a model with preselected regions and connections between them. It is based on the idea that temporal precedence is a characteristic that discerns causes from effects and uses a definition of causality for stochastic time series proposed by the economist Clive Granger [8,9]. Assigned to fMRI time series, the definition says that if activity in a voxel $x$ causes activity in a voxel $y$, then knowledge about the time series (activity over time) in voxel $x$ should help to explain future values of the time series in voxel $y$. If the future time series of $y$ is better explained by including $x$ than without $x$, given that all other useful information has also been included, we say that $x$ Granger causes $y$.

Granger causality maps (GCMs) were computed from signal time courses to estimate the directed influences between the ROI voxels and the other voxels in the scanned volume. For computation, the average time course of an ROI was taken as a reference and was considered as a potential target of influence from voxels from the rest of the brain as well as a potential source. Therefore, maps were computed showing voxels that are influenced by the activity in the ROI (Ref2Vox) and other maps showing

Table 1

Numbers of subjects in which Granger causality (negative GCM values) was found from bilateral auditory cortex and/or SMA to the ROIs (left/right motor cortex, SMA related to right/left motor response) in the group of 11 subjects

\begin{tabular}{|c|c|c|}
\hline \multicolumn{3}{|c|}{ Granger causality from predefined regions } \\
\hline \multirow[t]{2}{*}{ Region } & \multicolumn{2}{|c|}{ Subjects (of 11) } \\
\hline & $P<.001$ & $P<.01$ \\
\hline \multicolumn{3}{|c|}{ Granger causality to left motor cortex } \\
\hline Left auditory cortex & 9 & 9 \\
\hline Right auditory cortex & 9 & 10 \\
\hline SMA & 9 & 10 \\
\hline \multicolumn{3}{|c|}{ Granger causality to right motor cortex } \\
\hline Left auditory cortex & 5 & 7 \\
\hline Right auditory cortex & 5 & 7 \\
\hline SMA & 6 & 7 \\
\hline \multicolumn{3}{|c|}{ Granger causality to SMA related to right motor response } \\
\hline Left auditory cortex & 7 & 8 \\
\hline Right auditory cortex & 7 & 8 \\
\hline \multicolumn{3}{|c|}{ Granger causality to SMA related to left motor response } \\
\hline Left auditory cortex & 8 & 8 \\
\hline Right auditory cortex & 7 & 7 \\
\hline
\end{tabular}




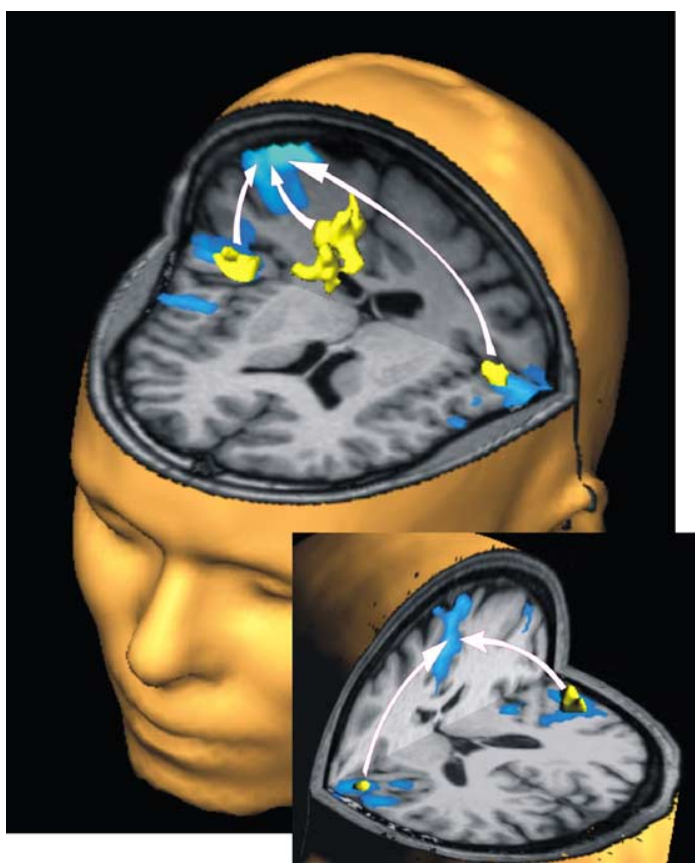

Fig. 1. Main picture: Granger causality from auditory cortex and SMA to motor cortex. Blue: Activation of auditory and motor cortex following the command "left" in a single subject thresholded at $P<.0001$ (uncorrected for multiple comparisons). Yellow: Three-dimensional GCM clusters in auditory cortex and SMA showing significant influence (above threshold negative values) to right motor cortex in the same subject thresholded at $P<.001$ (uncorrected for multiple comparisons). Small picture: Granger causality from auditory cortex to SMA. Blue: Activation of auditory cortex and SMA following the command "left" in a single subject thresholded at $P<.0001$ (uncorrected for multiple comparisons). Yellow: Three-dimensional GCM clusters in auditory cortex showing significant influence (above threshold negative values) to SMA in the same subject thresholded at $P<.001$ (uncorrected for multiple comparisons).

voxels whose activity influences the activation in the ROI (Vox2Ref). A third type of map showed voxels with instantaneous nondirected correlation (Inst). To obtain an unbiased estimate of directed influence, we computed the difference of Ref2Vox and Vox2Ref, showing positive values for influence from reference to voxel and negative values for the reverse direction [7]. We were interested to find voxels influencing our ROI voxels in motor cortex and SMA. Therefore, the difference map (Ref2Vox-Vox2Ref) with motor cortex or SMA as a reference was supposed to show negative values within those voxels. Bootstrapping [10] was used to assign significance to the GCMs [7]. Significant clusters in the GCMs were only accepted when they lay within or showed overlaps with the respective individual auditory/SMA activation of the subject and lay within the required anatomical region (auditory cortex, BA41; SMA, medial BA6) according to Talairach and Tournoux [5]. According to our hypothesis, activation within auditory cortex (voxel) Granger causes motor and SMA activation (reference), and SMA activation (voxel) Granger causes motor activation (reference); we only tested for the negative GCM values.

\section{Results}

Subjects showed high accuracy in the motor task. Nine subjects made no mistakes; 2 subjects made one mistake in pressing the ball with the correct hand after the command. One subject pressed the ball with the wrong hand in $25 \%$ of the trials and was therefore excluded from further analyses. Mean reaction times were $951.3 \mathrm{~ms}$ (S.D.: $223.6 \mathrm{~ms}$ ) for the right and $950.9 \mathrm{~ms}$ (S.D.: $251.2 \mathrm{~ms}$ ) for the left hand.

Granger causality maps of the single subjects centered on the individual motor cortex and SMA activation were analyzed with respect to the influences from the rest of the brain to the reference regions (Table 1). In 9 of 11 subjects, influence maps to the left motor cortex were significant at a level of .001 (uncorrected for multiple

Table 2

Brain areas besides a priori hypothesized regions (auditory cortex, SMA) from which Granger causality (negative GCM values) was found to the ROI (left/right motor cortex, SMA related to right/left motor response) and number of subjects (whole group: 11) in which the respective causality was found

Granger causality from brain areas not a priori hypothesized

No. of subjects Brain area

Granger causality to left motor cortex

1 or 2 Anterior cingulate cortex, medial prefrontal cortex (BA32/9), premotor cortex (BA6), thalamus, dorsal striatum, dorsal cingulate cortex, inferior temporal gyrus (BA37), medial temporal gyrus (BA21),

fusiform gyrus, precuneus, superior parietal lobe (BA7)

3 or $4 \quad$ Dorsolateral prefrontal cortex (BA9/46), superior temporal gyrus (BA22), occipital cortex 6 Putamen

Granger causality to right motor cortex

1 or 2 Dorsolateral prefrontal cortex (BA9/46), anterior cingulate cortex, medial prefrontal cortex (BA32/9), premotor cortex (BA6), thalamus, dorsal striatum, ventral striatum, superior temporal gyrus (BA22), inferior temporal gyrus (BA20), fusiform gyrus

3 or 4 Putamen, superior parietal lobe (BA7), occipital cortex

Granger causality to SMA related to right motor response

1 or 2 Anterior cingulate cortex, premotor cortex (BA6), dorsal cingulate cortex, superior temporal gyrus (BA22), medial temporal gyrus (BA21), inferior temporal gyrus (BA37), fusiform gyrus, precuneus, superior parietal lobe (BA7), occipital cortex

3 or 4 Dorsolateral prefrontal cortex (BA9/46), medial prefrontal cortex (BA32/9), dorsal striatum, putamen, thalamus,

Granger causality to SMA related to left motor response

1 or 2 Prefrontal cortex (BA45), premotor cortex (BA6), medial temporal gyrus (BA21), precuneus, superior parietal lobe (BA7), inferior parietal lobe (BA40)

3 or 4 Dorsolateral prefrontal cortex (BA9/46), medial prefrontal cortex (BA32/9), dorsal striatum, superior temporal gyrus (BA22), fusiform gyrus, occipital cortex,

5 Anterior cingulate cortex, thalamus

$7 \quad$ Putamen

$P<.001$; minimum cluster size: $50 \mathrm{~mm}^{3}$. 
comparisons) in bilateral auditory cortex as well as in SMA. At a level of .01 (uncorrected), influence maps to left motor cortex were significant in 10 subjects in right auditory cortex and SMA. Significant Granger causality to the right motor cortex existed at a level of .001 from bilateral auditory cortex in 5 and from SMA in 6 subjects. At a level of .01 significance, Granger causality to the right motor cortex existed from SMA and bilateral auditory cortex in 7 of 11 subjects. Fig. 1 shows auditory and motor activity and clusters Granger causing motor activity in a representative subject.

In 7 of 11 subjects, influence maps to the SMA activation related to the right motor response were significant at a level of .001 (uncorrected) in bilateral auditory cortex. At a level of .01 (uncorrected), influence maps to the SMA related to the right motor response were significant in 8 subjects in bilateral auditory cortex. Significant Granger causality to the SMA related to the left motor response existed at a level of .001 and also .01 from the left auditory cortex in 8 and from the right auditory cortex in 7 subjects.

Only subjects with above threshold negative GCM values (voxel to reference) are reported. The other subjects commonly showed below threshold negative values; none showed above threshold positive values in the hypothesized regions.

To provide further validation of our findings, we listed the other brain regions from which we found Granger causality to the four ROIs (negative, above threshold values of GCMs) in Table 2. Most of the regions were found inconsistently in only one or two subjects. The most consistent finding of Granger causality from other than hypothesized brain areas (auditory cortex and SMA) was Granger causality from the basal ganglia.

\section{Discussion}

Analysis of data with Granger causality in a standard event-related fMRI paradigm was able to demonstrate that the time course in motor cortex and SMA can be predicted by past signal time courses in areas that are known (by experimental design) to causally influence the two regions. Evidence for directed connectivity from auditory cortex and SMA to motor cortex was clearer for left motor cortex corresponding to the dominant hand than for right motor areas. Thus, the GCM analysis seems to imply a plausible chain of processing from auditory cortex to supplementary motor cortex to primary motor cortex in the investigated task. However, we should note that conclusions about chains of processing from GCMs should be drawn with care. Granger causality maps, in their current implementation, assess the influence between two regions independently of any others. In cases of an intervening role for a third area or common input from a third area, such influence might be spurious. In that case, investigating the GCM for the third area can avoid inferences of spurious direct influences [7]. As we used a relatively long TR, our findings show that even under suboptimal circumstances, the method can be a useful tool to explore effective connectivity. Besides from the a priori hypothesized areas, auditory cortex and SMA, we only found Granger causality consistently from basal ganglia. As basal ganglia trigger motor responses, this further supports the reliability of our findings.

The GCM method is applied in the face of the hemodynamic response that integrates the original neural activity and on temporal sampling of the data by the MR scanner. Granger causality in the context of fMRI is based on the existence of time lags of the hemodynamic response within brain areas representing causal influence of one region exerting on another (causes precede effects in time). But the time lags between different regions may occur for many other external reasons. The simplest are different characteristics of hemodynamic mechanisms in different brain regions. This might be a constraint of the method and could lead to wrong conclusions if the resulting maps are not interpreted with some care. There are two principle ways of dismissing hemodynamics as a principle cause of measured fMRI Granger causality. First, one could argue that although considerable variability in the (delay of the) hemodynamic response has been observed between different regions in the same brain (see, e.g., Ref. $[11,12]$ ), relative timing differences between BOLD responses could be used to discern a causal chain of cognitive processing across several areas $[13,14]$. Particularly, if variability in relative timing differences between the BOLD responses in areas involved in a given task can be attributed to task effects (e.g., relative timing differences correlate with reaction times), the GCMs likely reflect neural processing delays. This has been shown [13] for a cued visuomotor reaction time task very similar to the auditory cued reaction time task investigated here. They could show a progress of activation from V1 to SMA to M1 (similar to the causal chain implied by the GCMs computed here), and they showed that relative BOLD latencies (particularly those between V1 and SMA) were highly correlated with reaction times. A second way of dismissing hemodynamics as a physiological cause of Granger causality is by suitably contrasting GCMs between different conditions. If differences can be found between GCMs computed for different conditions, effective connectivity is found to be modulated by cognitive context and the influences found are more likely to reflect neurocognitive differences than hemodynamics. For instance, in this experiment, it would have been interesting to separate computation of GCMs for left-hand and right-hand responses and to show how influences are modulated by the response hand. However, the intermixed event-related design did not allow this, as experimental periods in which only left-hand and only right-hand responses were given were very short. Computing the GCMs over whole runs, pooling left-hand and right-hand responses, thus gave the expected result of aggregated influence of auditory cortex to both right and left motor cortices. 
Another important constraint for our design focused here is the temporal sampling of the data represented by the TR. Temporal precedence can only be detected if the time lag is big enough relative to the TR, otherwise, causality will fall into the instantaneous term and the method loses sensitivity for relatively fast directed influences. Roebroeck et al. [7] demonstrated the dependency of the sensitivity to detect interactions on the sampling interval (TR). The flow of information in cortical networks during certain cognitive operations might result in much faster sequences than a simple reaction time task. Therefore, short TRs resulting in a better sampling of the data are recommended. Still, we found reliable results with a TR of $2.5 \mathrm{~s}$. This shows that, dependent on the task, the method is not restricted to designs allowing short TRs but can be a promising approach for a wider variety of applications.

\section{Conclusion}

Our findings in a simple task show that even under suboptimal circumstances (a relatively long TR), Granger causality can be a useful tool to explore effective connectivity. Temporally optimized scanning should increase that potential.

\section{References}

[1] Buchel C, Friston K. Assessing interactions among neuronal systems using functional neuroimaging. Neural Netw 2000;13:871-82.
[2] McIntosh AR, Gonzalez-Lima F. Structural equation modeling and its application to network analysis in functional brain imaging. Hum Brain Mapp 1994;2:2-22.

[3] Friston KJ, Buchel C. Attentional modulation of effective connectivity from V2 to V5/MT in humans. Proc Natl Acad Sci U S A 2000;97:7591-6.

[4] Goebel R, Roebroeck A, Kim DS, Formisano E. Investigating directed cortical interactions in time-resolved fMRI data using vector autoregressive modeling and Granger causality mapping. Magn Reson Imaging 2003;21:1251-61.

[5] Talairach J, Tournoux P. Co-planar stereotaxic atlas of the human brain. Stuttgart: Thieme; 1988.

[6] Worsley KJ, Friston KJ. Analysis of fMRI time-series revisitedagain. Neuroimage 1995;2:173-81.

[7] Roebroeck A, Formisano E, Goebel R. Mapping directed influence over the brain using Granger causality and fMRI. Neuroimage 2005; $25: 230-42$.

[8] Granger CWJ. Investigating causal relations by econometric models and cross-spectral methods. Econometrica 1969;37:424-38.

[9] Granger CWJ. Testing for causality: a personal viewpoint. J Econ Dyn Control 1980;2:329-52.

[10] Efron B, Tibshirani R. An introduction to the bootstrap. New York: Chapman \& Hall; 1993.

[11] Aguirre GK, Zarahn E, D'Esposito M. The variability of human, BOLD hemodynamic responses. Neuroimage 1998;8:360-9.

[12] Miezin FM, Maccotta L, Ollinger JM, Petersen SE, Buckner RL. Characterizing the hemodynamic response: effects of presentation rate, sampling procedure, and the possibility of ordering brain activity based on relative timing. Neuroimage 2000;11:735-59.

[13] Menon RS, Luknowsky DC, Gati JS. Mental chronometry using latency-resolved functional MRI. Proc Natl Acad Sci U S A 1998; 95:10902-7.

[14] Formisano E, Goebel R. Tracking cognitive processes with functional MRI mental chronometry. Curr Opin Neurobiol 2003;13: $174-81$. 